\title{
Cooperation of axisymmetric connection elements under dynamic load
}

\author{
Andrzej Kołodziej ${ }^{1 *}$, Marian Dudziak $^{1}$, Krzysztof Talaśka ${ }^{2}$ \\ ${ }^{1}$ The President Stanisław Wojciechowski State University of Applied Sciences in Kalisz, Kalisz, \\ 62-800 Poland \\ ${ }^{2}$ Chair of Basics of Machine Design, Poznan University of Technology, Poznan, 60-965, Poland
}

\begin{abstract}
The article presents a method for determining the parameters that define the cooperation of the elements in the axisymmetic connection. The connection, which constitutes a shaft cooperating with a sleeve, has been tested for reaction forces in the connection during shaft rotation in the static sleeve. The shaft was characterized by deliberately modelled roundness deviations in the form of ovality, triangularity and quadrangularity. In addition, the research programme has taken into account the determination of the impact of tolerance of the outside diameter of the shaft. Determination of reaction forces has been carried out using the FEM software. The shaft has been modelled as a rigid element that rotates with a given rotational speed in the deformable sleeve. The conclusions present the impact of roundness deviation types and the tolerance value on reaction forces in the connection restraint. The method presented in the article can be used to predict the behaviour of the elements of axisymmetic connections under dynamic load, which can contribute to forecasting the durability of the connection.
\end{abstract}

Keywords: axisymmetric connections, dynamic loads, shape deviations

\section{Introduction}

Axisymmetric connections are some of the most basic connections in machine design. They come in two basic types: resting and movable. In the case of resting connections, their load carrying capacity is related to allowable stresses at rest. Movable connections are more vulnerable to damage, especially through wear and tear $[1,2]$. This is related to friction from the contact of interacting surfaces. The key in this kind of phenomena is the state of interacting surfaces It directly affects a number of tribological phenomena, leading to accelerated wear and tear of the connection. In the case of interacting cylindrical surfaces of the shaft and sleeve (outer and inner), what is also important apart from surface roughness, are shape deviations. This applies both to shape deviations of the cylinder generatrix (we can distinguish here: conicity, barrel-shapedness and saddle-shapedness) and to roundness deviations (ovalness, triangularity, quadriangularity, multiangularity) [1, 2]. When analysing production processes of the axisymmetric connection components, from a statistical point of view, it is possible to achieve any combination of the above mentioned

\footnotetext{
*Corresponding author: a.kolodziej@pwsz.kalisz.pl

Reviewers: Grzegorz Domek, Marek Macko
} 
shape deviations. Figure 1 shows sample results of measuring shafts with detected cross section shape deviations (multiangularity). It is possible, of course, to control the production process in order to achieve selected shape deviations with higher probability. In order to do it successfully, given the expected life cycle of the connection, methods of determining the parameters of interaction between such connections in operating conditions corresponding to dynamic loads should be developed. An example would be rotational movement of the shaft relative to the sleeve. It was decided that the main parameter defining the nature of the axisymmetric connection would be the value of the reaction force at fixed support during shaft rotation in the hub.
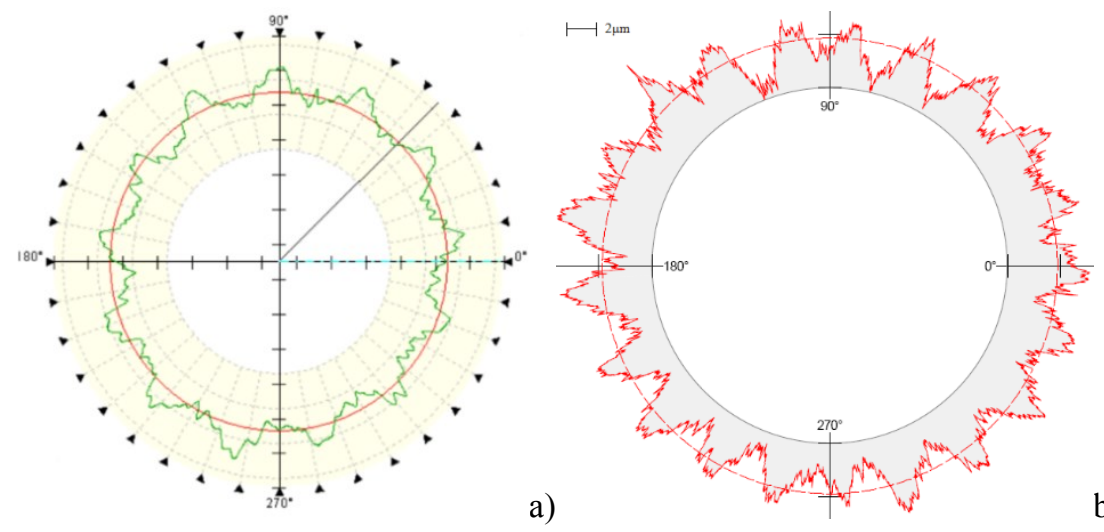

Fig. 1. a) 13-angularity chart, b) 21-angularity chart [7]

The finite element method was used for the above mentioned analyses. Model shafts characterized by saddle-shapedness and ovalness, triangularity and quadriangularity were analysed. Cross section shape deviations were made in four tolerance grades IT2, IT4, IT6 and IT9.

\section{Computational model creation}

Figure 2 shows the stages of computational model creation. Simplifications were used, based on symmetry in the vertical axis and by using a small surface contact area between the shaft and the sleeve. As a result, the issue was reduced to the flat problem $1 \mathrm{~mm}$ thick (this value of the contact between the outer shaft surface and the inner sleeve surface was assumed). The entire process of creating the computational model is analogous to the one presented in the works [3-7]. Figure 3 shows the types of studied shape deviations (diameter $ø 19 \mathrm{~mm}$ ), including ovalness, triangularity and quadriangularity in four tolerance grades: IT2 $(2.5 \mu \mathrm{m})$, IT4 $(6 \mu \mathrm{m})$, IT6 $(13 \mu \mathrm{m})$ and IT9 $(52 \mu \mathrm{m})$. The shaft length was assumed as $70 \mathrm{~mm}$. In the FEM model (Abaqus software), it was assumed that a fixed shaft rotates with the specified rotational speed (1 RPS, 10 RPS and 100 RPS) in a deformable sleeve. Figure 4 shows the FEM model. Material properties: Young modulus E $=210 \mathrm{GPa}$, Poisson's ratio $v=0.3$, density equal to $7830 \mathrm{~kg} / \mathrm{m}^{3}$. Considering the simplification of the model, it was loaded in such a way as if the entire shaft with the assumed geometric parameters (without additional load) rotated in the sleeve. The first stage of the work with the model was to determine the minimum number of finite elements on the external surface of the sleeve. During calculations, this surface was fixed and from this place the values of reaction forces were recorded. The stages of minimising the number of finite elements are presented in Figure 4. 

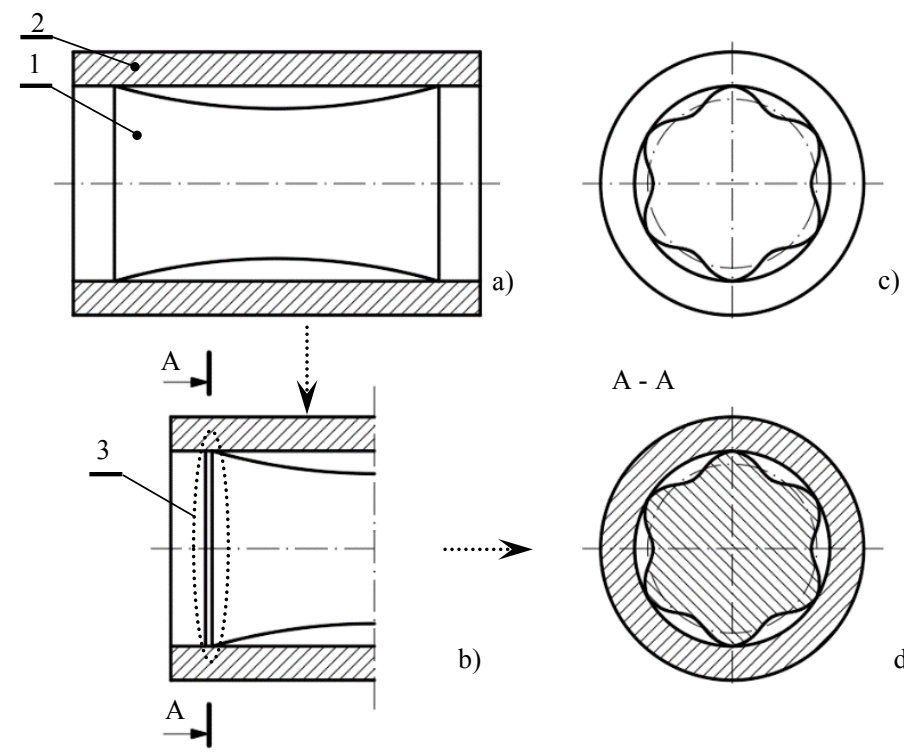

A - A

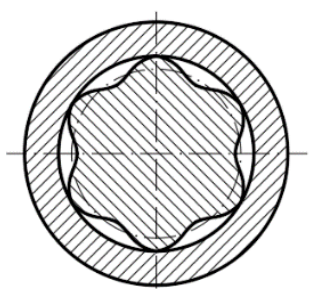

d)

Fig. 2. Stages of computational model creation: a) real model: 1 - saddle shaft, 2 - nominally round hub, b) simplification using symmetry relative to the vertical axis: 3 - shaft-hub contact area,

c) simplification to the flat problem thanks to a short length of the shaft where shaft-hub contact takes place [7]
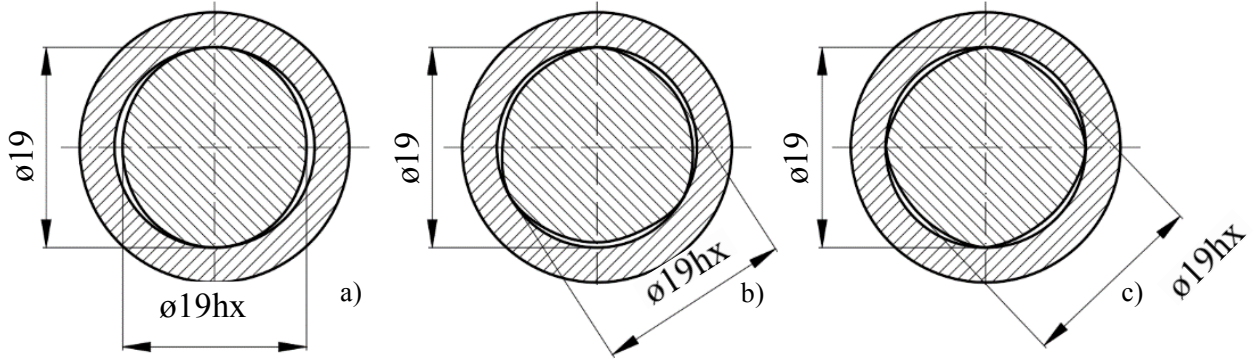

Fig. 3. Studied shape deviations: a) ovalness, b) triangularity, c) quadriangularity, $x$ - tolerance grade, grades IT2, IT4, IT6, IT9 were analysed
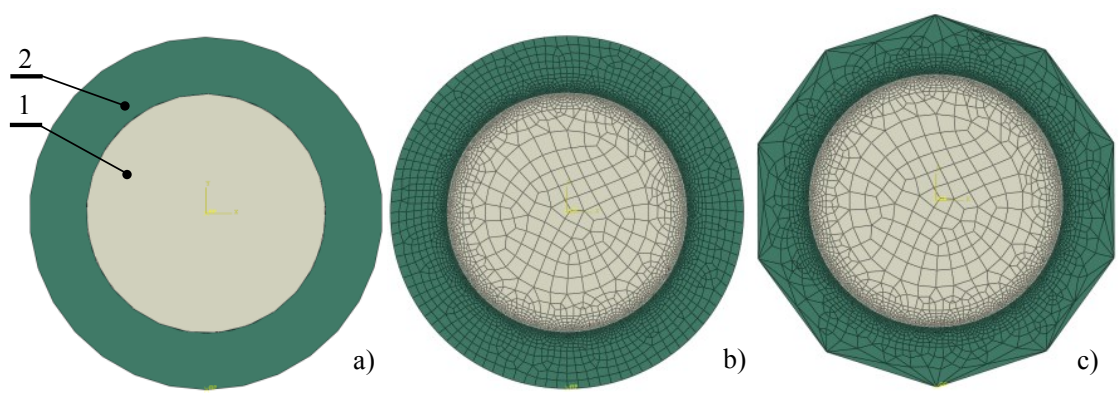

Fig. 4. FEM model: a) flat model: 1 - shaft, 2- sleeve, b) discretized model with 100 finite elements on the outer surface of the sleeve, c) discretized model with 10 finite elements on the outer surface of the sleeve 
Figure 5 shows charts with the values of reaction force in the sleeve fixed support for 100 elements on the outer surface of the sleeve (fig. 5a) and with 10 elements on the outer surface of the sleeve (fig. 5b). Calculations were made for the same gravity force, these values were compared with gravity force computed analytically. It can be clearly seen that a decrease in the number of finite elements on the outer surface does not adversely affect the accuracy of the calculations. This is the basis for the simplification of the model.
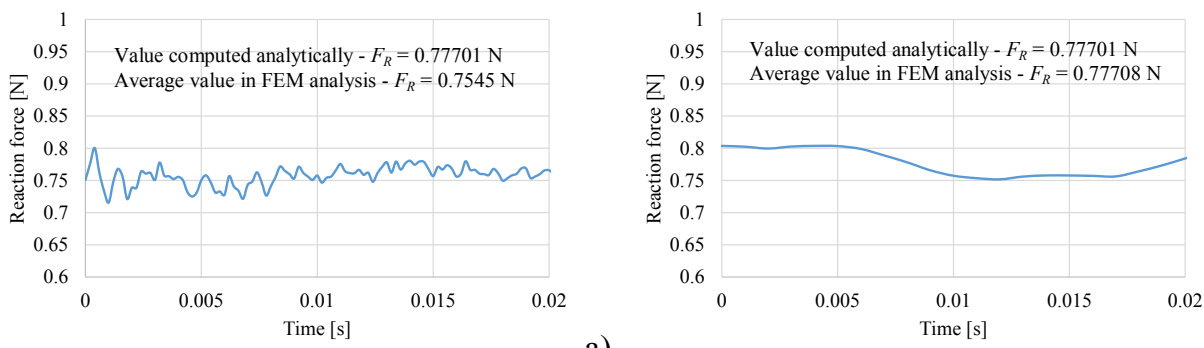

a)

Fig. 5. Reaction force $F_{R}$ : a) from the analysis performed on the model with 100 elements on the outer surface of the sleeve, $b$ ) from the analysis performed on the model with 10 elements on the outer surface of the sleeve

\section{Simulation results}

Figure 6 shows sample stress distributions in the sleeve in selected angular positions of the shaft (oval) relative to the sleeve. Figure 7 and 8 present the same results for a triangular and quadriangular shaft. Stress distributions are only for reference, the purpose of the charts is to indicate contact zones of the shafts with shape deviations, which is why tables with stress values are not included. Figures 9-11 present the change in the value of reaction force $F_{R}$ in fixture for the oval shaft made in the 9th tolerance grade for three speeds (one rotation). A marked increase in the value of reaction force is visible. Figures 12-15 present the change in the value of reaction force $F_{R}$ for the triangular shaft made in four difference tolerance grades (rotational speed 1 RPS). Figures 16 and 17 present distributions of reaction force $F_{R}$ for the triangular shaft (IT9) for rotational speed 10 RPS and 100 RPS. Figures 18-21 present the change in the value of reaction force $F_{R}$ for the quadriangular shaft made in four difference tolerance grades (rotational speed 1 RPS). Figures 22 and 23 present distributions of reaction force $F_{R}$ for the quadriangular shaft (IT9) for rotational speed 10 RPS and 100 RPS.

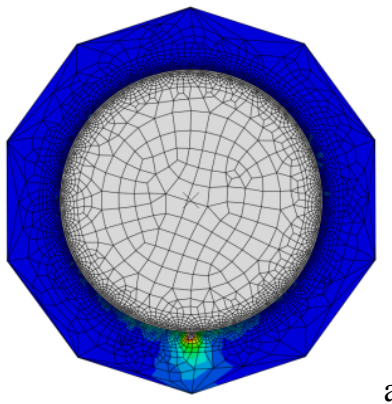

a)

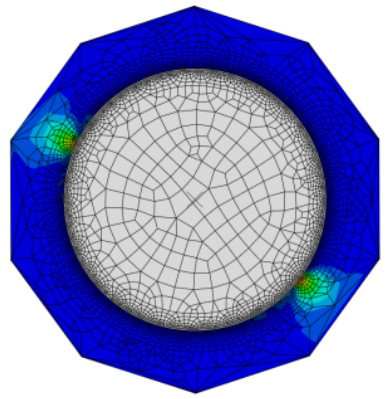

b)

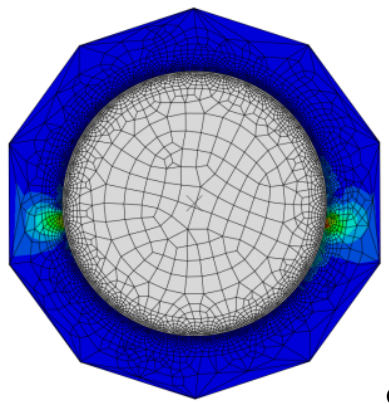

c)

Fig. 6. Sample stress distributions for an oval shaft: a) position $0^{\circ}$, b) position $22.5^{\circ}$, c) position $45^{\circ}$ 

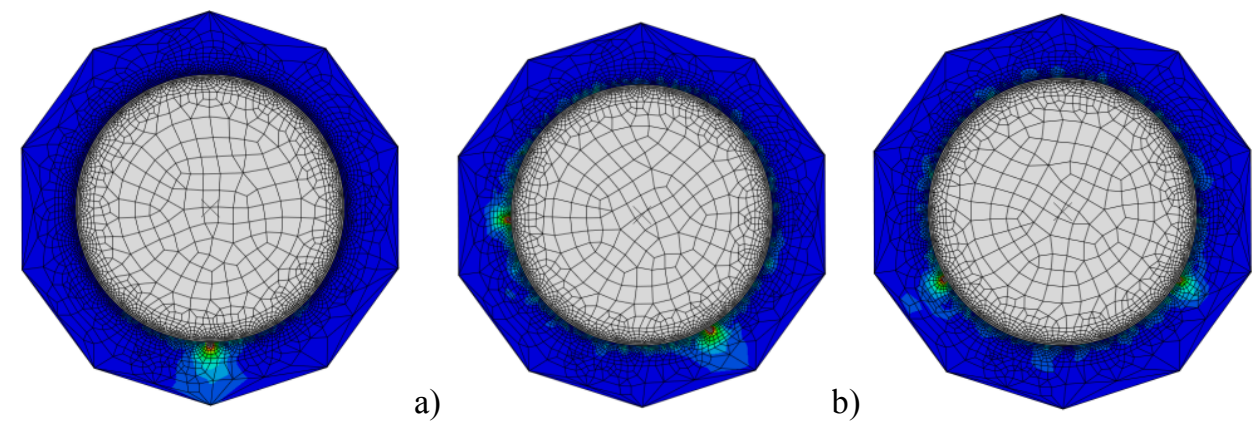

c)

Fig. 7. Sample stress distributions for a triangular shaft: a) position $0^{\circ}$, b) position $30^{\circ}$, c) position $60^{\circ}$
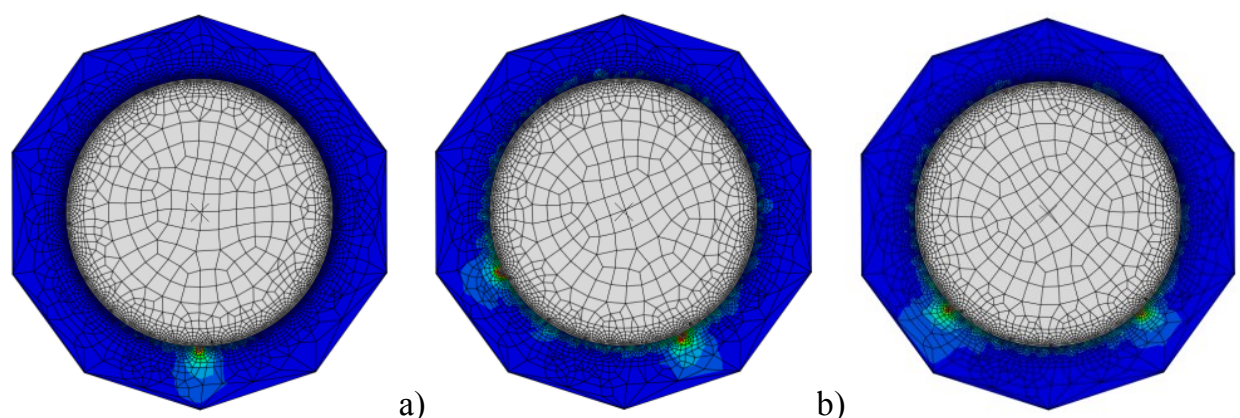

Fig. 8. Sample stress distributions for a quadriangular shaft: a) position $0^{\circ}$, b) position $22.5^{\circ}$, c) position $45^{\circ}$

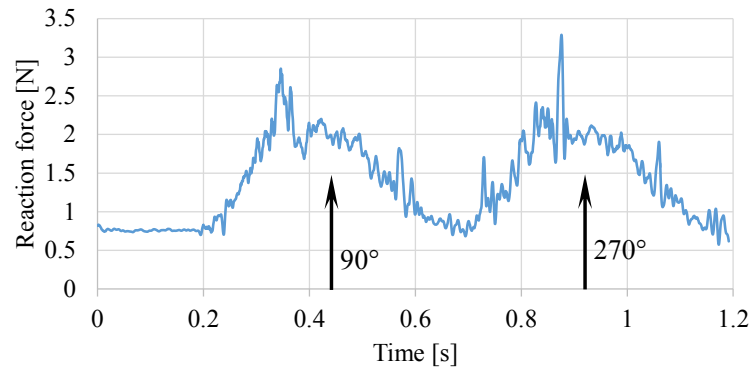

Fig. 9. Reaction force in the sleeve fixture: oval, IT9, 1 RPS

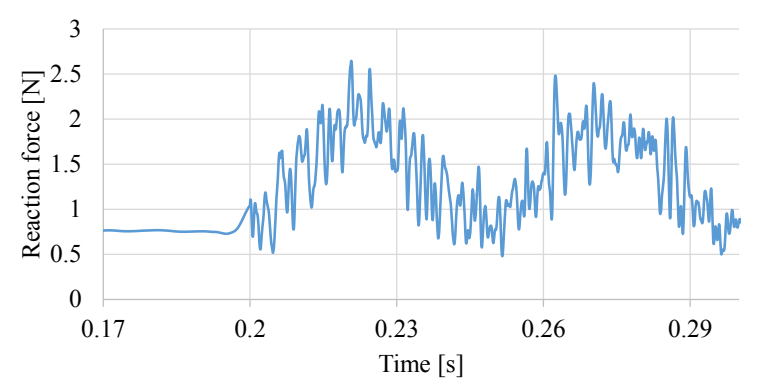

Fig. 10. Reaction force in the sleeve fixture: oval, IT9, 10 RPS 


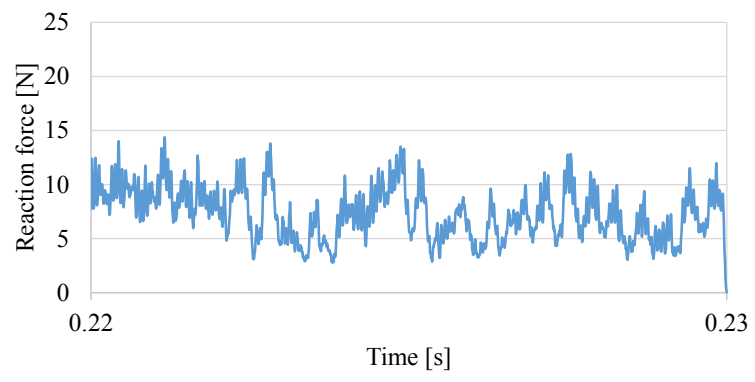

Fig. 11. Reaction force in the sleeve fixture: oval, IT9, 100 RPS

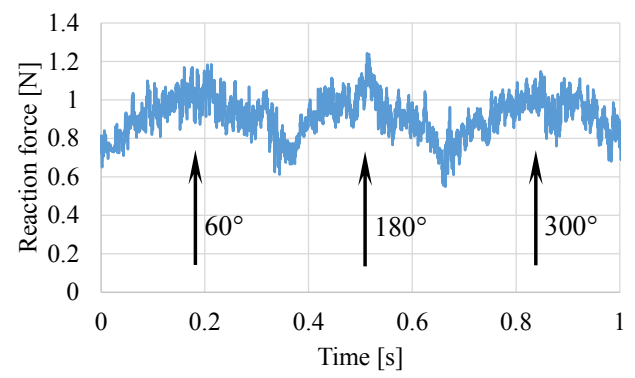

Fig. 12. Reaction force in the sleeve fixture: triangularity, IT2, 1 RPS

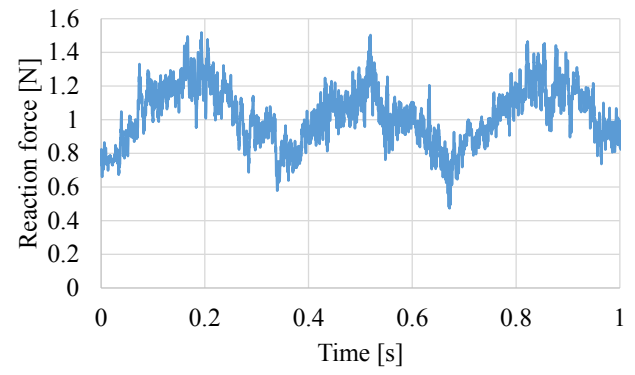

Fig. 13. Reaction force in the sleeve fixture: triangularity, IT4, 1 RPS

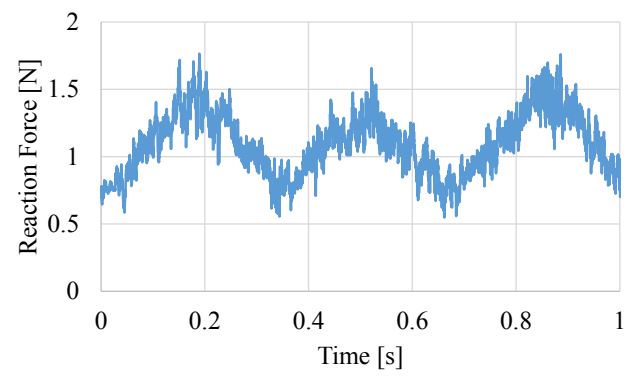

Fig. 14. Reaction force in the sleeve fixture: triangularity, IT6, 1 RPS 


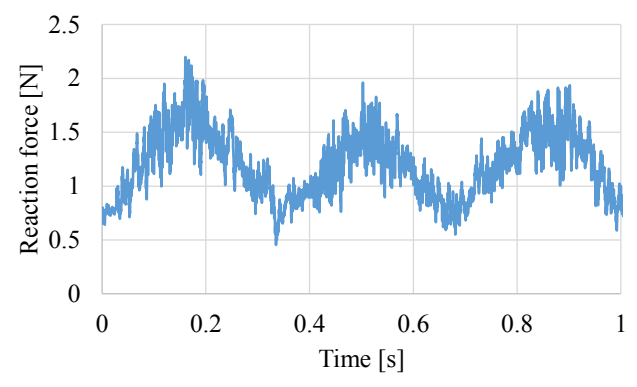

Fig. 15. Reaction force in the sleeve fixture: triangularity, IT9, 1 RPS

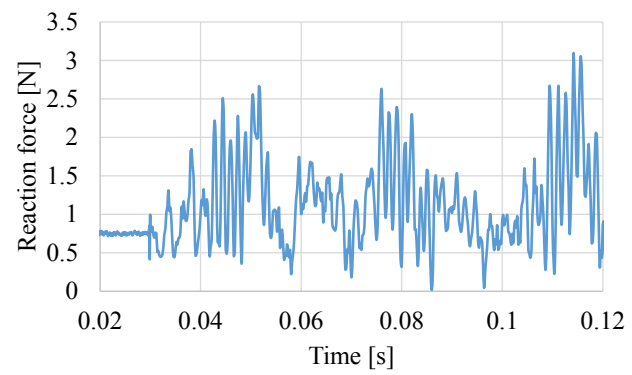

Fig. 16. Reaction force in the sleeve fixture: triangularity, IT9, 10 RPS

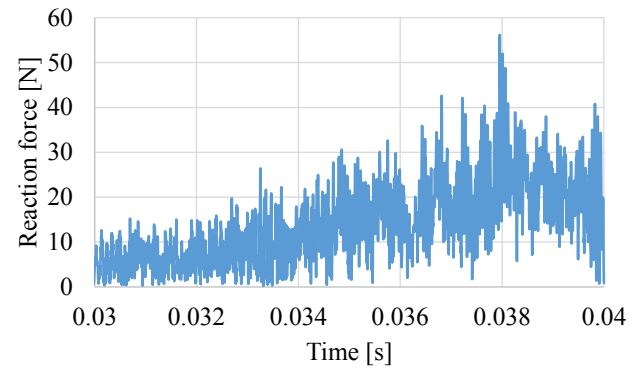

Fig. 17. Reaction force in the sleeve fixture: triangularity, IT9, 100 RPMS

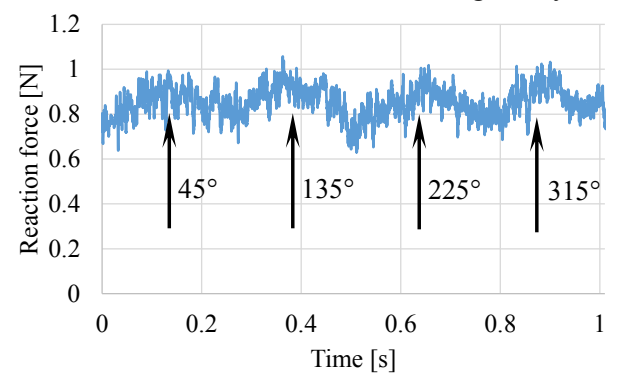

Fig. 18. Reaction force in the sleeve fixture: quadriangularity, IT2, 1 RPS 


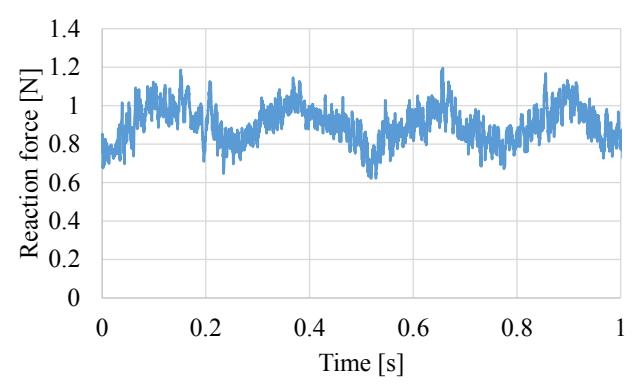

Fig. 19. Reaction force in the sleeve fixture: quadriangularity, IT4, 1 RPS

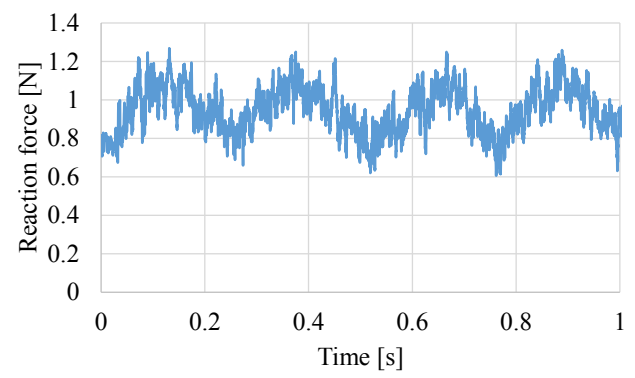

Fig. 20. Reaction force in the sleeve fixture: quadriangularity, IT6, 1 RPS

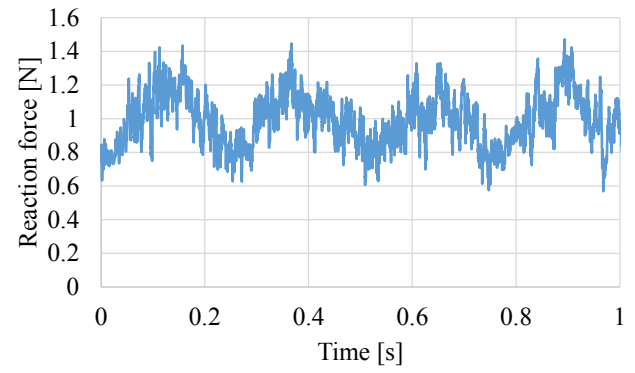

Fig. 21. Reaction force in the sleeve fixture: quadriangularity, IT9, 1 RPS

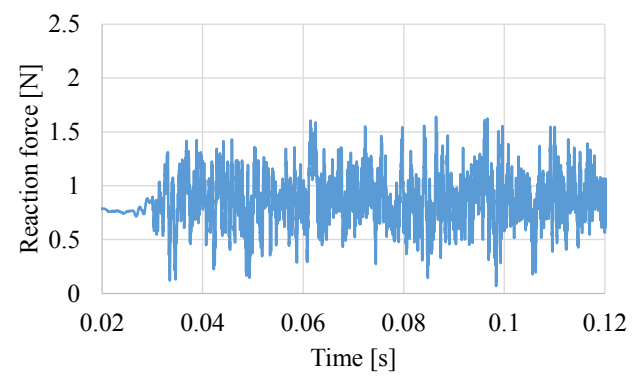

Fig. 22. Reaction force in the sleeve fixture: quadriangularity, IT9, 10 RPS 


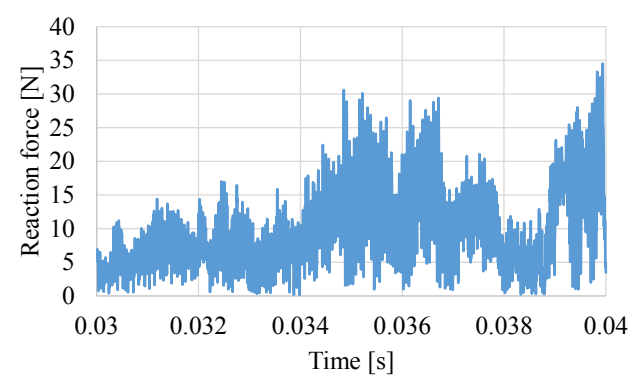

Fig. 23. Reaction force in the sleeve fixture: quadriangularity, IT9, 100 RPS

\section{Conclusions}

The method for determining the course and changes in the value of reaction forces $F_{R}$ in the sleeve fixture of the axisymmetric connection with shape deviations presented in this work is the approach to the problem proposed by the authors. Analysing the course of reaction forces $F_{R}$ it can be easily stated that both shape deviations and tolerance grade of the connection components have a significant impact on the value of the presented forces.

It is important that the actual cross-sectional shape of the shaft affects the nature of the changes in the value of the reaction force $F_{R}$. The identification of these forces and their analysis can contribute to better knowledge of the phenomenon of vibrations of machine systems equipped with the axisymmetric connection. The increasing rotational speed of the shaft relative to the sleeve (especially for inferior tolerance grades) unambiguously leads to a significant increase in the value of the reaction force $F_{R}$. It is quite an obvious conclusion but at the same time it confirms high usability of the presented method. The next stage of this research should be to check the impact of additional connection load on the characterization of cooperation parameters.

\section{References}

1. S. Adamczak, Geometrical measurements of surface - shape, waviness and roughness. (WNT, Warsaw, 2008)

2. S. Adamczak, W. Makieła, K. Stępień, Investigating advantages and disadvantages of the analysis of a geometrical surface structure with the use of Fourier and wavelet transform. Metrology and Measurement Systems XVII, 233-244 (2010)

3. M. Dudziak, G. Domek, A. Kołodziej, Variation of static parameters of cooperation in axisymmetric connection. Procedia Engineering 136, 56-62 (2016)

4. M. Dudziak, G. Domek, A. Kołodziej, K. Talaśka, Contact Problems Between the Hub and the Shaft with a Three-angular Shape of Cross-section for Different Angular Positions. Procedia Engineering 96, 50-58 (2014)

5. M. Dudziak, A. Kabała, A. Kołodziej, The Topic of Contact Zone Problems in the Shaft and Hole Joint, Taking into Account Component Form Errors. Journal of Mechanics Engineering and Automation 3, 586-590 (2013)

6. M. Dudziak, G. Domek, A. Kołodziej, K. Talaśka, Contact Problems Between the Hub and the Shaft with a Four-Angular Shape of Cross-Section for Different Angular Positions. Applied Mechanics and Mechatronics II, Applied Mechanics and Materials, 54-62 (2015)

7. M. Dudziak, G. Domek, A. Kołodziej, K. Talaśka, Multi-angularity - identification of 
parameters and compatibility conditions of the axisymmetric connection with form deviations. Procedia Engineering 177, 431-438 (2017) 